\title{
Cardiovascular Urgencies and Emergencies in Pregnancy: A Case Based Review of Some Cardiovascular Diseases Affecting Pregnant Females
}

\author{
Timothy Paterick*
}

Department of Cardiology, Aurora Health Care, USA

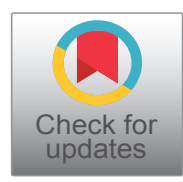

*Corresponding author: Timothy Paterick, Professor of Medicine, Department of Cardiology, Aurora Health Care, USA

\begin{abstract}
This manuscript explores the clinical presentation and physical examination findings in pregnant patients with presumed heart disease. There is a review of the clinical presentation, physical examination, and multi-modality imaging that led to a diagnosis in pregnant patients with coronary artery disease, cardiomyopathies, arrhythmias, valvular heart disease, and pulmonary hypertension. The cases are not exhaustive, but represent a subset of the large number of cardiovascular conditions affecting pregnant patients. For an excellent and exhaustive review, the readership is directed to the 2018 ESC Guidelines for the management of cardiovascular disease during pregnancy.
\end{abstract}

\section{Keywords}

Pregnancy, Coronary dissection, Amniotic fluid embolism, Pulmonary embolism, Bicuspid aortic valve and aortopathy, Cardiomyopathy, STEMI, Pulmonary hypertension, Takotsubo cardiomyopathy, Ventricular arrhythmias, Postpartum cardiomyopathy

\begin{abstract}
Abbreviations
SCAD: Spontaneous Coronary Dissection; TC: Takotsubo Cardiomyopathy; PE/AFE : Pulmonary Embolism/ Amniotic Fluid Embolism; PPH: Primary Pulmonary Hypertension; PPC: Post-Partum Cardiomyopathy; VT: Ventricular Tachycardia; DCM: Dilated Cardiomyopathy; BAVIA: Bicuspid Aortic Valve with Aortopathy; HF: Heart Failure; GDMT: Guideline Directed Medical Therapy; A/C: Anticoagulation; SC: Supportive Care; A/Ab/D: Antiarrhythmic/Ablation/Device; FH: Family History; G: Genetics; RM: Rhythm Monitoring
\end{abstract}

\section{Methods Section}

This case-series of cardiovascular diseases presenting in pregnant females explored the medical approach to acute cardiovascular presentations in pregnant females. The cases were reviewed retrospectively through an indepth exploration of the medical records. Individual patients were contacted by telephone and asked if their case could be published if anonymity was maintained. All living patients agreed. The deceased patient's mother agreed.

The case reviews allowed for highlighting of the clinical presentations, physical examination findings, imaging features and treatment approaches to patients with spontaneous coronary artery dissection versus paradoxical embolism to the coronary tree, stress induced cardiomyopathy, pulmonary embolism versus amniotic fluid embolism, primary pulmonary embolism, peripartum cardiomyopathy, ventricular arrhythmias, dilated cardiomyopathy, and bicuspid aortic valve associated aortopathy (Table 1 ).

\section{Introduction}

The potential cardiovascular diseases in pregnancy are vast as detailed in the excellent 2018 ESC Guidelines for the management of cardiovascular disease in pregnancy. The recognition and management of cardiovascular disease impacting the health of pregnant females and their baby is daunting. The pregnant female is susceptible to hypertension/pre-eclampsia, benign to life-threatening arrhythmias, cardiomyopathies, heart failure, valve disease, coronary artery disease, aortopathy, congenital heart disease, pulmonary hypertension, and venous disease. 
Table 1: Results/cases section: Assuming excellent medical care.

\begin{tabular}{|l|l|l|l|l|l|}
\hline CASE & DISEASE & INCIDENCE & DIAGNOSIS & TREATMENT & PROGNOSIS \\
\hline 1 & SCAD & Rare & Cath & Medical vs. PCI & Good \\
\hline 2 & TC & Rare & Echo/Cath & GDMT for HF & Good \\
\hline 3 & PE/AFE & Rare & Echo/CT & A/C: SC & Good/Guarded \\
\hline 4 & PPH & Low & Echo & Vasodilators & Poor \\
\hline 5 & PPC & Low & Echo & GDMT & Good/Guarded \\
\hline 7 & VT & Rare & RM & A/Ab/D & Guarded \\
\hline 8 & DCM & Rare & FH/G/Echo & GDMT/A & Poor \\
\hline
\end{tabular}

SCAD: Spontaneous Coronary Dissection; TC: Takotsubo Cardiomyopathy; PE/AFE: Pulmonary Embolism/Amniotic Fluid Embolism; PPH: Primary Pulmonary Hypertension; PPC: Post Partum Cardiomyopathy; VT: Ventricular Tachycardia; DCM: Dilated Cardiomyopathy; BAVIA: Bicuspid Aortic Valve With Aortopathy; HF: Heart Failure; GDMT: Guideline Directed Medical Therapy; A/C: Anticoagulation; SC: Supportive Care; A/Ab/D: Antiarrhythmic/Ablation/Device; FH: Family History; G: Genetics; RM: Rhythm Monitoring

It is essential for the practicing cardiologist to have an in-depth understanding of the diseases and treatment approaches. The 8 cases discussed in this manuscript just touch the surface of cardiovascular conditions physicians may encounter in daily clinical practice. This manuscript will hopefully stimulate the readership to further study the vast array of cardiovascular conditions potentially affecting pregnant patients.

The cases discussed below provide examples of some complex clinical conditions faced by the cardiologist when called to see the pregnant patient with symptoms suggesting cardiovascular disease. These cardiovascular conditions require rapid recognition and actions to prevent fetal and maternal morbidity and mortality. These are casing the author encountered in his daily clinical practice.

For each case there is an analysis of the clinical presentation and physical examination signs prompting recognition of cardiovascular disease impacting the patient's health. There is an elucidation of the pathophysiology of the cardiovascular abnormality, detailing of how imaging modalities facilitate making the cardiac diagnosis. Multi-modality imaging is often the linchpin to diagnosis. These cases are designed to stimulate the readership to pursue a deeper understanding of the cardiovascular illnesses impacting pregnant patients. The 2018 ESC Guidelines for the management of cardiovascular disease during pregnancy give an all-encompassing review of cardiovascular disease management in pregnant patients.

\section{Cardiovascular Urgencies and Emergencies}

\section{Case 1}

Cardiology was consulted on a 32-year-old female for severe shortness of breath (SOB), chest pressure, and labile hemodynamics during delivery. She had two prior normal deliveries.

Post-delivery she required intubation and had a blood pressure (BP) of 190/100 $\mathrm{mm} \mathrm{Hg}$ and heart rate
(HR) of 130 beats per minute (bpm). The ECG revealed sinus tachycardia, low voltage QRS and poor R wave progression with diffuse, non-specific ST changes (Figure 1).

Treatment with a diuretic, beta-blocker, and intravenous nitroprusside resulted in a BP $=150 / 90$ and $\mathrm{HR}=95 \mathrm{bpm}$. Oxygenation was adequate with assisted ventilation. Urgent echocardiography revealed left ventricular (LV) systolic function was depressed with an ejection fraction of $20 \%$, dilated right ventricle (RV) with severe reduction of RV systolic function, severe tricuspid regurgitation (TR) and elevated right heart pressure (Figure 2). Incidentally, an atrial septal defect (ASD) was identified.

Urgent cardiac tomography (CT) scan was negative for pulmonary embolism (PE) and aortic dissection. Magnetic resonance (MR) imaging revealed the left ventricular ejection fraction (LVEF) was $22 \%$ and the RV was enlarged. The differential diagnosis generated was a stress-induced cardiomyopathy, paradoxical embolism to the coronary tree, and spontaneous coronary artery dissection. The patient became hemodynamically stable with medical therapy and was extubated with normal oxygen saturations.

Follow up evaluation of the ASD with a transesophageal echocardiography (TEE) revealed a thin, dyskinetic distal anterior septum and apex, a sinus venous ASD, and normal LV and RV systolic function. Right heart pressure was normal. The patient was feeling well without symptoms and baby was doing well.

Review of the case raised a question of an apical myocardial infarction due to SCAD versus embolism to the coronary tree. This led to review of the prior ECGs. The ECG analysis supported the notion of an anterior infarct, as the initial ECG was normal (Figure 3).

The ECG post-delivery revealed loss of $R$ waves across the precordium (Figure 1). This retrospectively raised a strong suspicion that the initial insult was 


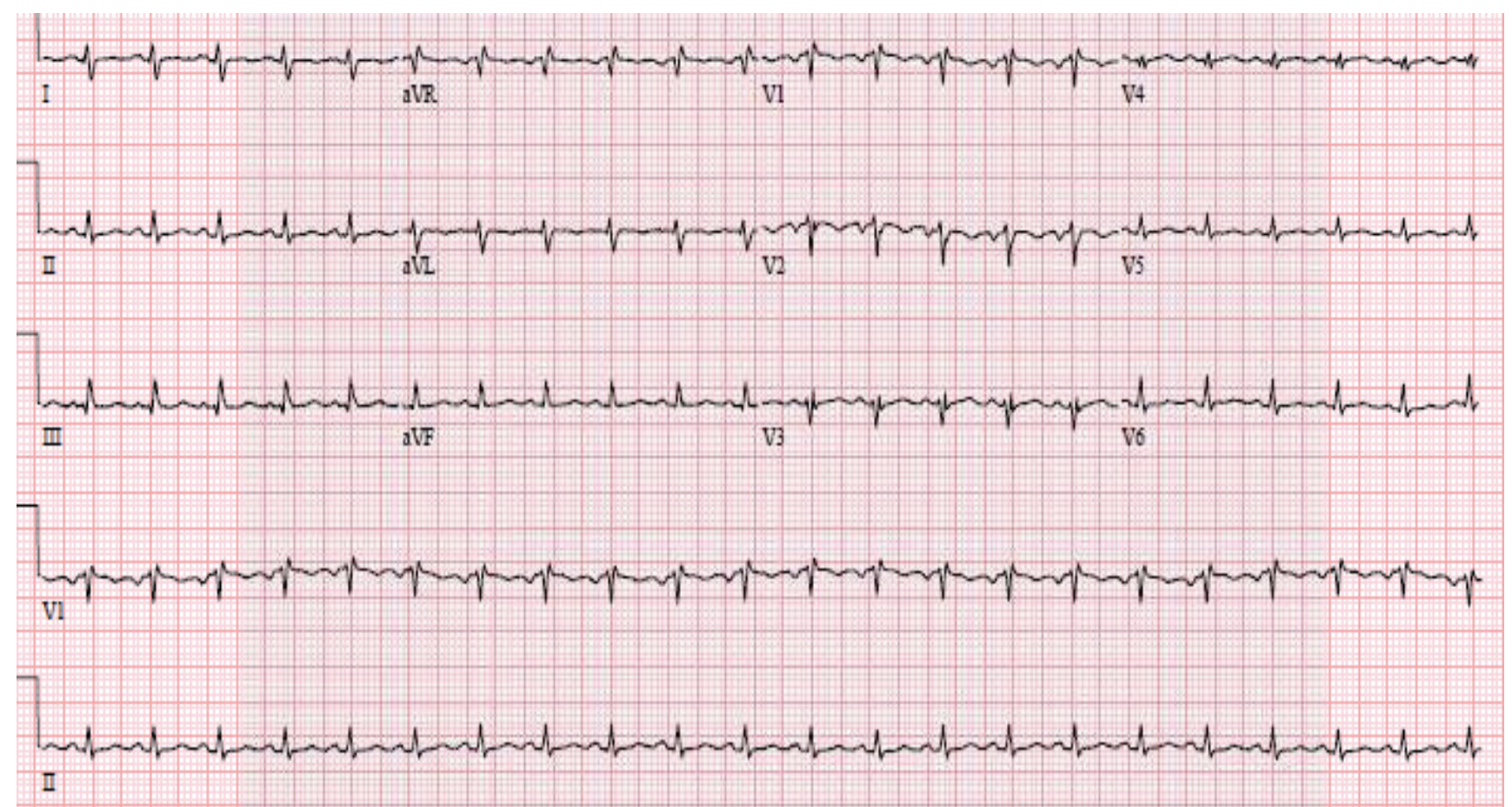

Figure 1: Sinus tachycardia with loss of $\mathrm{R}$ waves across the precordium and non-specific ST changes.

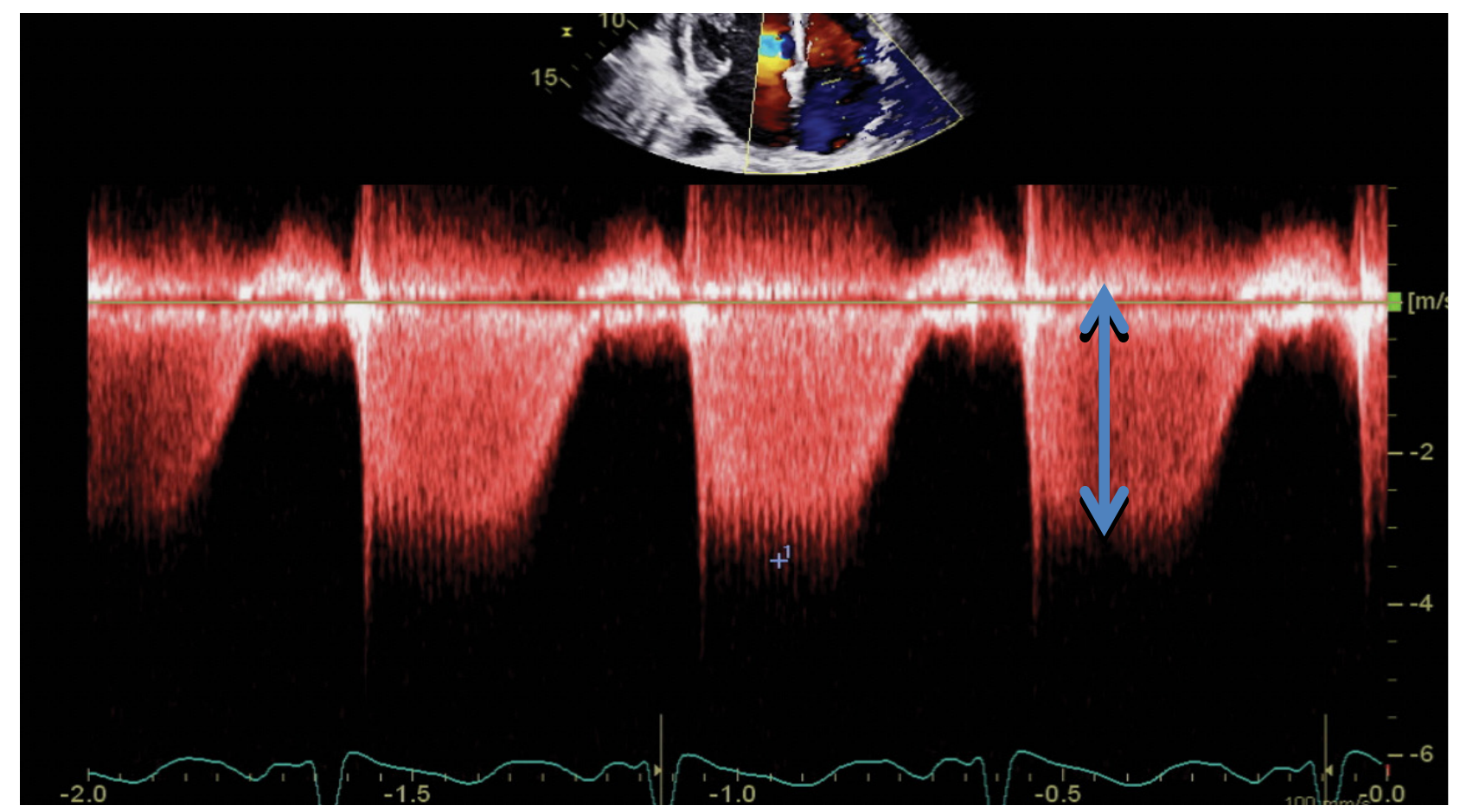

Figure 2: TR envelope: Blue arrow to $3.5 \mathrm{~m} / \mathrm{sec}$. The RV-RA gradient is approximately $50 \mathrm{mmHg}$.

an unrecognized coronary artery dissection versus a paradoxical embolism across the ASD to the coronary circulation. The interventional team felt the angiogram was supportive of a dissection. This infarct was supported on MRI and TEE imaging. Surgery was consulted for evaluation of the sinus venous ASD in the setting of enlarged right heart chambers by echocardiography and MRI. The review of the MRI revealed normal venous connections. Because of the apical infarct, surgery requested a cardiac catheterization. The catheterization revealed an occluded left anterior descending coronary artery (Figure 4). This occluded vessel retrospectively identified the etiology of the post-delivery hemodynamic instability. The patient was feeling well and elected not to pursue surgery for the ASD or coronary disease.

Pregnancy and spontaneous coronary artery dissection (p-scad): P-SCAD is a rare condition with an incidence of $(0.1 \%)$ for all patients referred for coronary angiography [1]. The mean age at presentation is 35-40 


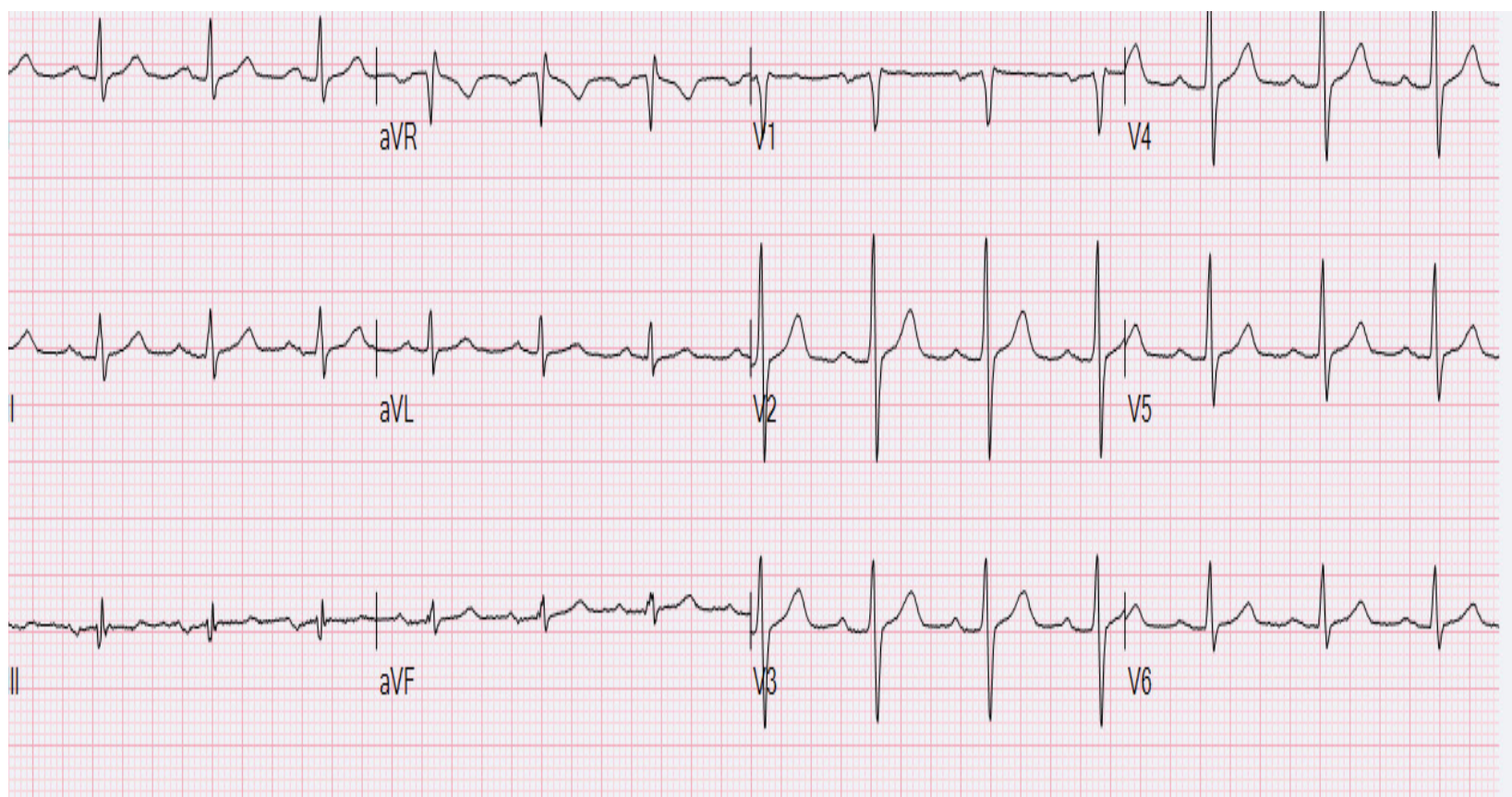

Figure 3: Normal ECG prior to delivery.

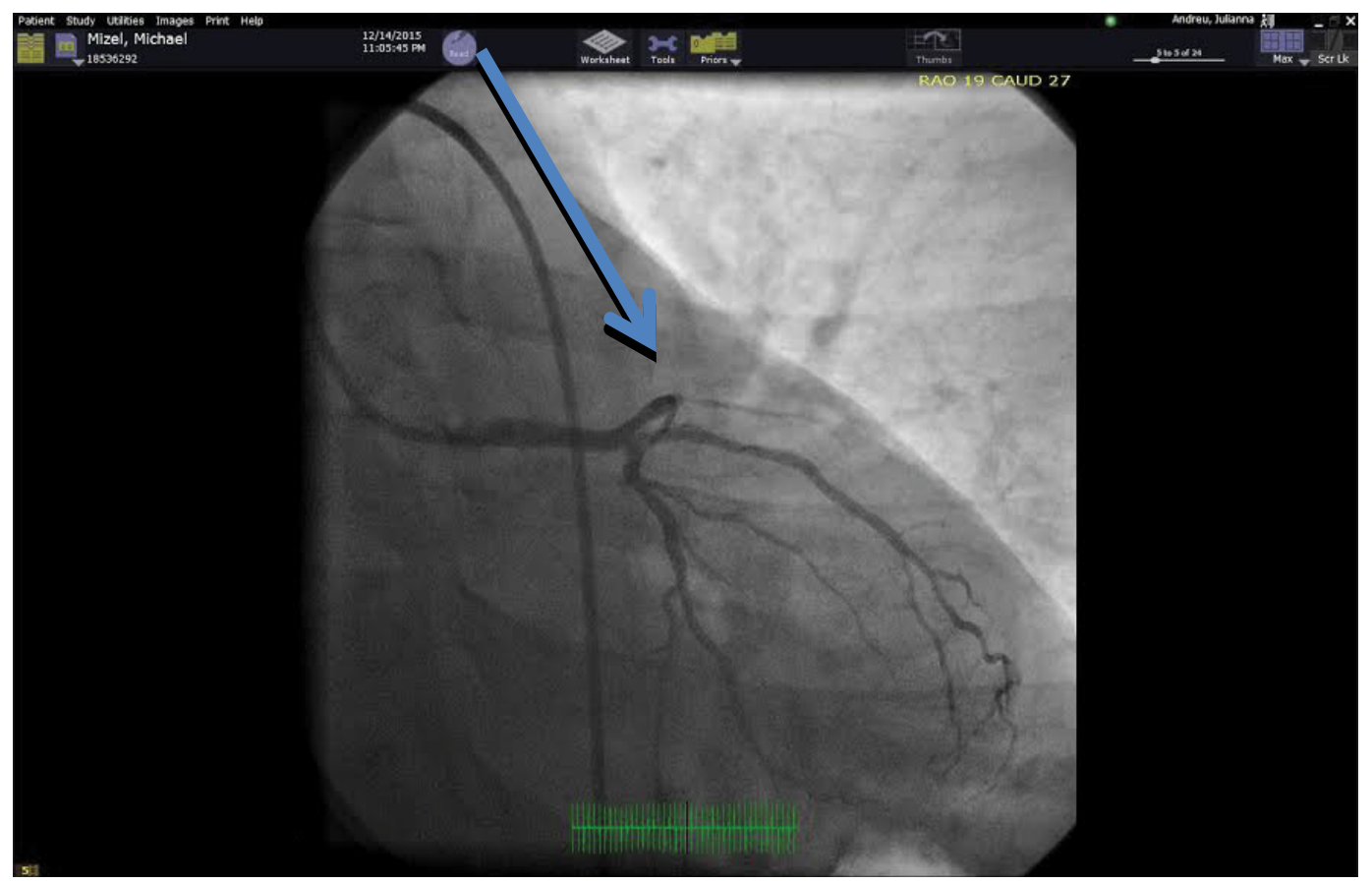

Figure 4: Coronary angiography reveals an occluded LAD (Blue arrow).

years, and greater than $70 \%$ of SCAD cases are female [2]. Typically, patients with spontaneous coronary artery dissection are divided into four etiologic groups: peripartum, atherosclerotic, idiopathic, and vasculitic in patients with connective tissue disease [2]. This classification does not cover all potential etiologies of SCAD.

Approximately (33\%) of P-SCAD cases occur in the peripartum period, with $1 / 3$ late in pregnancy and $2 / 3$ in the early puerperal period [2]. The peak incidence is two weeks after delivery. The role of the peripartum period in the pathogenesis is an enigma. Theories include hormonal changes, such as high estrogen levels, resulting in subtle changes in arterial wall architecture with ensuing susceptibility to spontaneous dissections. These changes include hypertrophy of the smooth muscle cells, loosening of the intracellular matrix due to increase in acid mucopolysaccharides, and decreased collagen production in the media $[3,4]$. Additionally, increased total blood volume, high cardiac output, and straining and shearing forces during labor may result in increased wall stress. Thus, hemodynamic and hormonal changes are thought to increase the risk of intimal tears. Patients in the pregnancy subset with underlying connective 


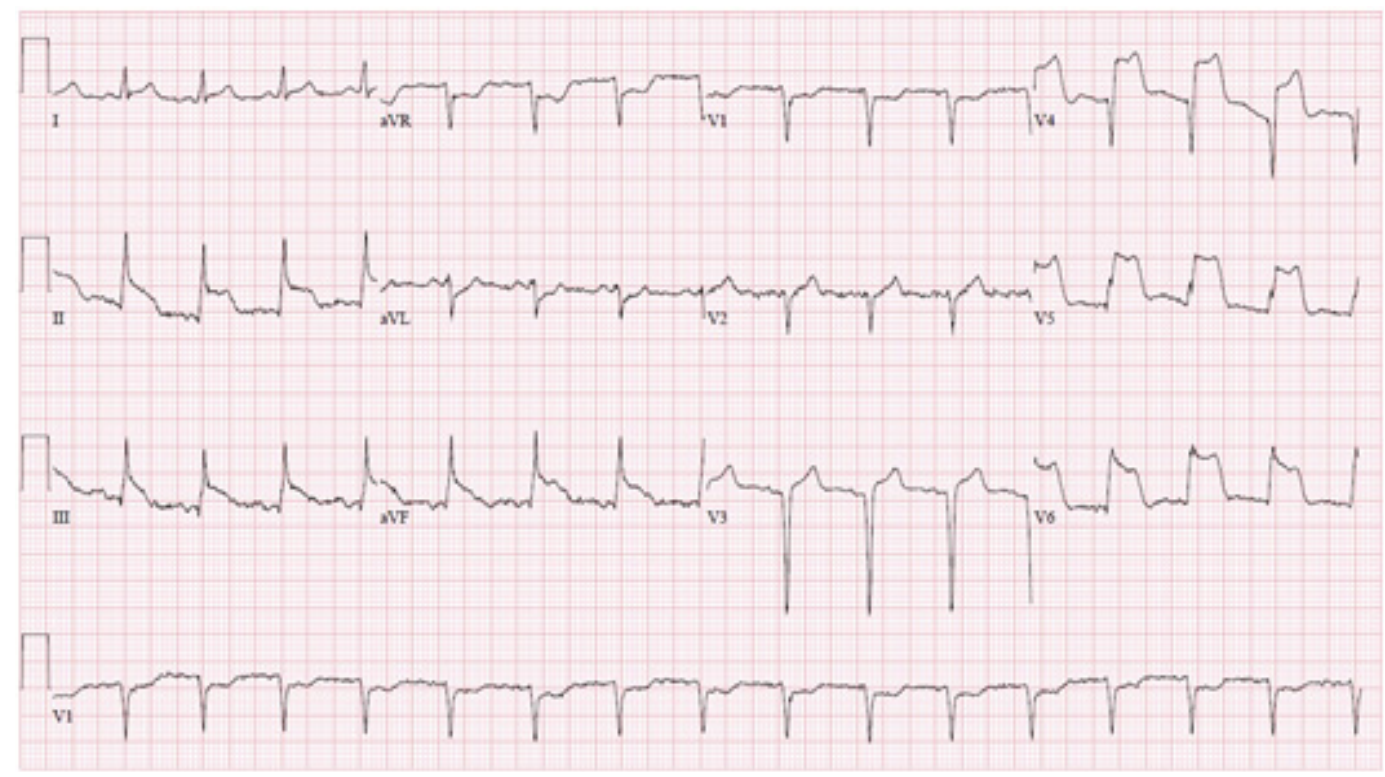

Figure 5: ECG revealing ST elevation inferiorly and laterally.

tissue disorders, such as Marfan syndrome, Ehler-Danlos type 4 and systemic lupus erythematous with vasculitis, may be at even higher risk for P-SCAD $[5,6]$.

P-SCAD is thought to be the consequence of an intramural hematoma of the coronary artery, resulting in a false lumen compressing the true lumen, resulting in myocardial ischemia [7]. The clinical presentation ranges from unstable angina to sudden cardiac death. Young woman with angina and ECG changes should lead to a high suspicion for SCAD and urgent evaluation and angiography should be considered. In young women the left anterior descending coronary artery is the most common location [2]. When angiography is performed the approach ranges from mostly conservative to rarely revascularization with stenting depending on the angiographic and clinical circumstances.

\section{Take home messages for P-SCAD}

- Awareness and recognition of P-SCAD is critical to the diagnosis. Acute myocardial infarction (AMI) in pregnancy should be treated similarly to AMI in the general population [8].

- A multidisciplinary approach between cardiology and obstetrics is mandatory as data is lacking regarding the safety of guideline directed medical therapy (GDMT) in pregnancy. Expert opinion is that low dose aspirin (ASA), beta-blocker (BB) therapy and short-term heparin during percutaneous intervention $(\mathrm{PCl})$ are reasonable therapies [8].

- The most experienced operators, due to the risk associated with $\mathrm{PCl}$, should undertake revascularization. Generally, coronary intervention should be limited to cases that are unstable. The approach to P-SCAD must be determined on a caseby-case basis by the multidisciplinary team [8].

\section{Case 2}

29-year-old female was in her sixth month of pregnancy. She had no prior personal or family history of cardiovascular disease. She had experienced a prior miscarriage. The miscarriage was emotionally draining and resulted in depression. Psychology consultation resulted in treatment for anxiety and depression.

Resting at home, she developed chest pain, nausea, and loss of consciousness. Emergency medical services were summoned and the paramedics performed an ECG revealing ST elevation inferolaterally (Figure 5). Upon arrival to the emergency room, she developed ventricular fibrillation requiring cardiac defibrillation to restore sinus rhythm.

In the intensive care unit, the patient was hemodynamically stable with an infusion of intravenous dobutamine. She was alert and cognitively intact. Urgent echocardiography revealed an akinetic apex with hypercontractile basal segments. LVEF was estimated to be $35 \%$. There was no associated outflow tract obstruction. There was mild mitral regurgitation. Urgent angiography revealed normal coronary arteries. Laboratory results revealed a mildly elevated troponin and normal electrolytes.

The patient was treated with a beta-blockers and diuretic. She clinically recovered without chest pain, shortness of breath, or further rhythm disturbance. Troponin levels normalized and the ECG evolved from ST elevation to inverted t waves inferolaterally. Repeat echocardiography on day 7 revealed normalization of the apical hypokinesis. LVEF $=60 \%$. The patient was discharged home and at nine months gave birth via caesarian section to a healthy baby without newborn cardiovascular deformity.

Takotsubo cardiomyopathy in pregnancy (P- 
TC): This case represents a rare form of P-TC with a malignant, life threatening rhythm disturbance. There is no available evidence that pregnancy predisposes to a stress-induced cardiomyopathy. The majority of stress induced cardiomyopathies recover to normal LV function. In patients where LV dysfunction persists, GDMT is the standard of care and the criteria for implantation of an implantable cardio-defibrillator are determined on a case-by-case basis $[9,10]$.

Takotsubo cardiomyopathy (TC) is a unique form of transient non-ischemic cardiomyopathy that typically occurs in a setting provoked by a stressor - physical, emotional, or both - and hence, is also given the nickname of stress-induced cardiomyopathy. The syndrome occurs most commonly in post-menopausal females. The characteristic clinical syndrome of TC involves acute LV dysfunction with distinctive echocardiographic feature of apical to mid ventricular hypo- to akinesis and sparing of the basal myocardium in the absence of significant obstructive coronary artery disease (CAD).

Alternatively, patients may exhibit reverse TC with basal hypokinesis and hyperkinesia of the apical and mid segments of the left ventricle [11]. Even though the apical ballooning phenotype is the most common and typical presentation, much confusion has resulted from various nomenclatures being used for different presentations of this syndrome.

A well-recognized syndrome now, it is also being reported in populations other than postmenopausal women. The occurrence in premenopausal women is rare, and a literature search reveals there are few cases reported in pregnancy [11].

Patients usually present with typical chest pain (70-90\%) and dyspnea (20\%); other less common presentations include syncope, pulmonary edema and cardiac arrest [10]. Dynamic electrocardiographic changes and elevated cardiac biomarkers (reflecting acute myocardial injury) are usually present [11]. Coronary angiography, however, typically does not reveal any evidence of epicardial coronary obstruction. However, patients with CAD can experience stressinduced cardiomyopathy. Symptoms can be severe and lead to death in $2 \%$ of patients [12]. Song and colleagues reported $32 \%(n=16)$ of their patients with TC $(n=$ 50) presented with cardiogenic shock as the initial presentation [1].

The most common electrocardiographic changes reported in TC are ST-segment elevations in precordial leads on admission (range, $46-100 \%$ of patients). Subsequent deep symmetrical T-wave inversion in multiple leads and Q-wave formation (range, 6-31\% of patients) are frequently found $[9,10]$. Also, QT interval prolongation (range, 450-501 ms) can be present $[8,13]$. The combination of clinical symptoms and electrocardiographic changes at patient's initial presentation makes differentiation of TC from ACS very challenging. Most TTC patients present with elevated cardiac biomarkers and have a modest peak in levels within 24 hours, but levels are markedly lower than would be anticipated on the basis of the extent of wall motion abnormalities and electrocardiogram findings [14-17].

Management focuses on supportive care in the acute phase, while avoiding vasopressor medications because hyperdynamic basal LV can result in outflow tract obstruction. Mortality is low if patients survive the initial critical period and, by definition, they go on to have a full recovery. Recurrence has been reported but recurrences are rare. TC follow-up revealed the rate of major adverse cardiac and cerebrovascular events were 9.9\% per patient year, and the rate of death was $5.6 \%$ per patient year [17].

\section{Take home messages for P-TC}

- $\mathrm{P}-\mathrm{TC}$ is very rare

- P-TC ventricular arrhythmias are rare

- Patients with P-TC presenting with an acute coronary syndrome (ACS) should be managed according to guidelines for the general population including revascularization techniques [8].

- P-TC patients presenting with heart failure (HF) should be treated according to HF guidelines. Management goals are similar to non-pregnant HF therapy, while avoiding medications toxic to the fetus including angiotensin converting enzyme (ACE) inhibitors, angiotensin 11 receptor blockers (ARBs), angiotensin - nephrilysin inhibitors (ARNI), aldosterone receptor antagonists (MRA) and atenolol. HF with congestion should be treated with loop diuretics, but avoided if feasible due to potential reduction in placental blood flow. Hydralazine and nitrates are safe in pregnancy, although less effective than ACE inhibitors, and should only be used in the presence of hypertension and LV dysfunction with or without congestion. Beta-blockers should be up- titrated starting at low dose to desired effect [8].

- Management of electrical disturbances in P-TC can include: cardioversion, as it is presumed safe in all stages of pregnancy as it does not compromise fetal blood flow. The risk of fetal arrhythmias or initiation of pre-term labor is low [8].

\section{Case 3}

25-year-old female presented to the emergency room with severe shortness of breath and hypoxia. She had delivered a full-term baby girl via caesarian section and was discharged five days previously. She was short of breath post-delivery and upon discharge. She became progressively more short of breath. Her chest 


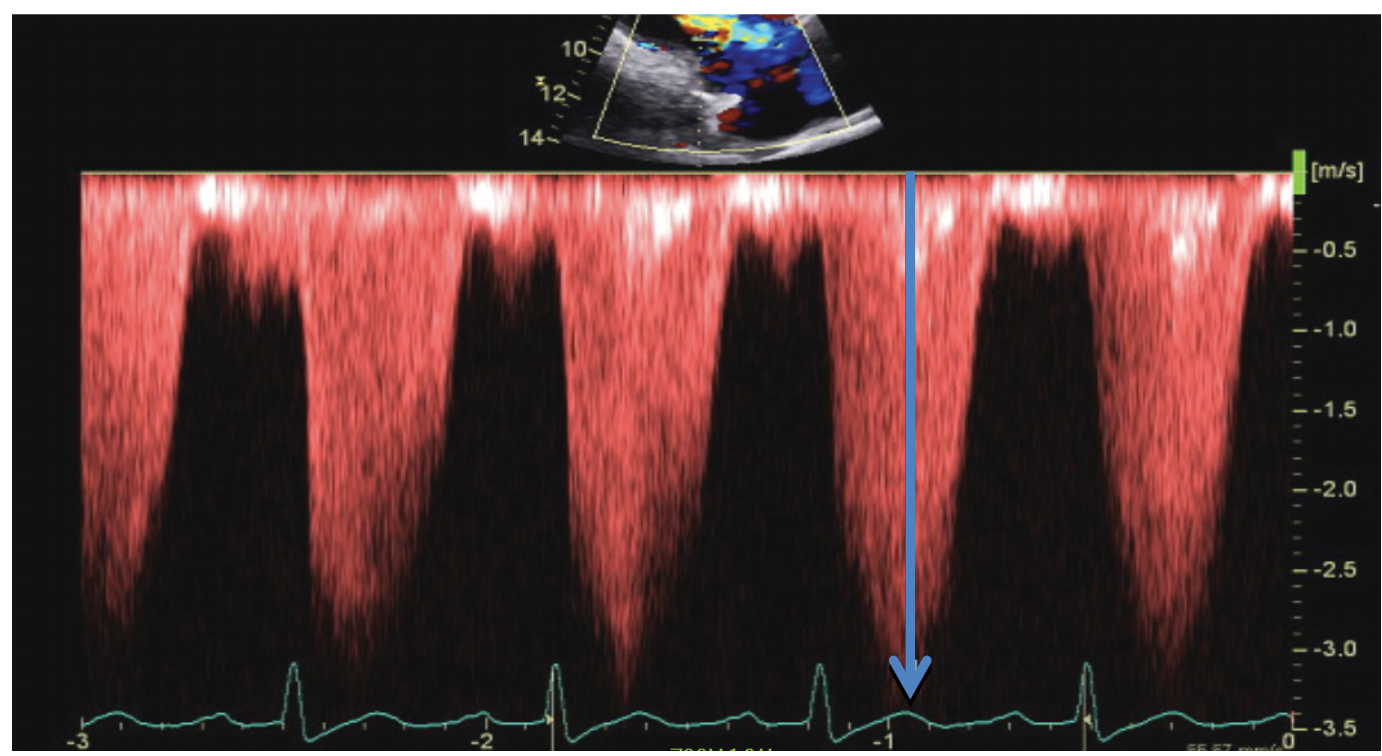

Figure 6: TR envelope is $3.35 \mathrm{~m} / \mathrm{sec}$. The RV - RA gradient + RAP $(10 \mathrm{mmHg}) \sim 55 \mathrm{mmHg}$

X-ray revealed diffuse alveolar infiltrates suggesting pulmonary edema.

Cardiology was consulted. She was hemodynamically stable with a blood pressure of $160 / 90$ and heart rate of $95 \mathrm{bpm}$. Respiratory rate was elevated at 20 per minute. Her jugular venous pressure (JVP) was elevated and she had a 2/4-holosystolic-murmur at right $4^{\text {th }}$ intercostal space. There was no leg edema.

An urgent echocardiogram revealed an enlarged RV with decreased systolic function with severe tricuspid regurgitation (TR) and estimated pulmonary artery systolic pressure (PASP) was $55 \mathrm{~mm} \mathrm{Hg}$ (Figure 6). LV systolic function was normal. Treatment included a beta-blocker, diuretic, and low dose angiotensinogen converting enzyme inhibitor. An urgent CT pulmonary angiogram was negative for pulmonary embolism. The working exclusionary diagnosis became amniotic fluid embolism (AFE).

She stabilized clinically with improved hemodynamics and respiratory status. She was discharged on medical therapy. In 6 weeks, she had a repeat echocardiogram revealing normal LV systolic function, decreased RV size, and mild TR with an estimated PASP $=25 \mathrm{~mm} \mathrm{Hg}$. The patient and the baby were both doing well.

Pulmonary embolism versus Amniotic Fluid Embolism in Pregnancy (P- PE/AFE): Pregnancy and the puerperium are well-established risk factors for venous thromboembolism and anaphylactic syndrome of pregnancy related amniotic fluid embolism. Both pulmonary embolism (PE) and amniotic fluid embolism (AFE) should be considered in a pregnant or post-partum patient with hypotension, hypoxia, hypertension, and right heart failure [18].

There are no clinical symptoms or signs that are specific for pulmonary embolism (PE). There is an overlap between symptoms of PE and normal physiologic changes of pregnancy [18]. Thus, identifying a clinically important PE during pregnancy, or post-partum, is challenging. It is essential that clinicians understand the potential for over diagnosing and under diagnosing PE during pregnancy. PE is a common cause of death in the hospital and should always be considered when pregnant patients have tachycardia and shortness of breath.

Diagnostic considerations include D-dimer levels, leg ultrasound imaging, and CT pulmonary angiography (CTPA). CTPA has high sensitivity and specify $(100 \%$, $89 \%)$ for detecting pulmonary emboli $[19,20]$. Rapid diagnosis leads to appropriate anticoagulation therapy and improved survival of the infant and mother. Undiagnosed PE has a mortality rate approaching $30 \%$, which is reduced to approximately $2-8 \%$ when diagnosed and treated appropriately [21,22].

When the CTPA is negative for a pulmonary embolism there should be thoughtful consideration for AFE. AFE is a rare and potential fatal obstetric emergency. AFE may occur during pregnancy, but is more commonly identified during labor and early post-partum. Risk factors for AFE include fetal distress, maternal age, placental abnormalities, eclampsia, polyhydramnios, cervical lacerations, and cesarean section, and rapid delivery. AFE is an allergic - like reaction to amniotic fluid entering the mother's circulation. Management is monitoring and treating the respiratory, cardiovascular, and hematological perturbations that arise. AFE is not preventable, but it is essential to recognize it and treat it expectantly [23].

\section{Take home message for P- PE/AFE}

- Pulmonary emboli (PE) and deep venous thrombosis (DVT) are known etiologies of pregnancy related morbidity and mortality. 
The risk of venous thromboembolism (VTE) is paramount in the immediate post-partum period and does not return to normal risks until 6 weeks post-delivery. A high index of suspicion and low threshold for investigation is imperative in pregnant patients [8].

- The optimum diagnostic approach for pregnant females is undefined. A Wells score in combination with D-dimer (low specificity in pregnant patients) testing is a strategy attempting to determine when imaging is needed. A negative D-dimer is helpful, but low D-dimer concentrations have been observed in normal pregnancies [8].

- If VTE suspicion is high, then compression ultrasonography should be performed and if negative, but suspicion remains high, then further testing with low-dose CT scanning should be performed [8].

- When the suspicion is high and testing is underway, the patient should be treated with low molecular weight heparin (LMWH). LMWH is the drug of choice for treatment of VTE in pregnancy and the puerperium [8].

- When CTPE is negative, AFE is the diagnosis of exclusion and careful monitoring is necessary with treatment of organ system complications [23].

\section{Case 4}

21-year-old primigravida female presented to the emergency room at 32 weeks of gestation. She had a 7-week history of progressive shortness of breath associated with central chest pressure and tachycardia. She had not sought prior medical care. She was unaware of any health issues.
On physical examination she had tachycardia at 110 bpm and tachypnea with a respiratory rate of 22 per minute. Blood pressure was 100/60. Jugular venous pressure was elevated to the angle of the jaw while sitting up. She had a right parasternal heave and lower extremity-pitting edema. Her second heart sound was accentuated. She had a pan-systolic murmur at the right sternal border.

The electrocardiogram revealed $P$ pulmonale, RAD, and RV strain pattern. The chest $X$-ray revealed prominent RV silhouette and dilated pulmonary arteries.

The echocardiogram revealed severe $\mathrm{RV}$ dilation and hypertrophy (RVH), moderate RV systolic dysfunction, a large right atrium (RA), severe $P R$, severe $T R$, plethoric IVC with a calculated right ventricular systolic pressure (RVSP) of $80 \mathrm{~mm} \mathrm{Hg}$ (Figure 7) and the plethoric inferior vena cava (IVC) revealed systolic flow reversal in the hepatic vein (HV). The mitral inflow Doppler and tissue Doppler were normal.

A duplex ultrasound of the lower extremities and a negative D-dimer suggested low probability for pulmonary embolic disease. Blood screening revealed no evidence of vasculitis or connective tissue disease.

The clinical diagnosis was primary pulmonary hypertension (PPH). The patient was started on a diuretic, sildenafil $50 \mathrm{mg}$ three times daily, and an intravenous infusion of heparin for two days prior to delivery.

An elective caesarian section under general anesthesia was performed with a pulmonary artery catheter and arterial line. Her systolic pulmonary artery pressure (PAP) was $100 \mathrm{~mm} \mathrm{Hg}$. She was intubated and ventilated. Nitric oxide was started after induction of anesthesia. A healthy baby was delivered. Nitric oxide

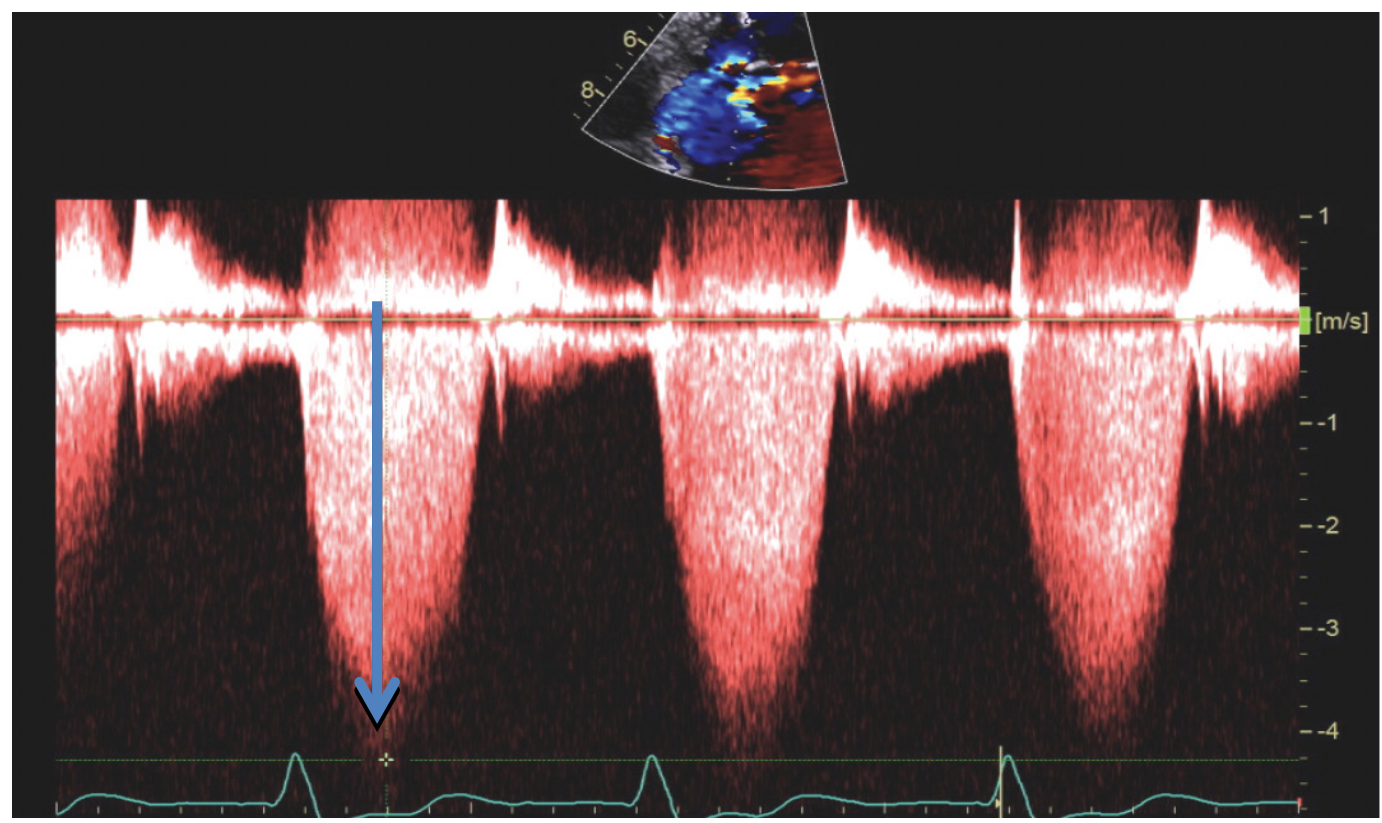

Figure 7: TR envelope $4.1 \mathrm{~m} / \mathrm{sec}$ (Blue arrow). RV-RA gradient + RAP $80 \mathrm{mmHg}$. 
was continued. The PAP was $90 \mathrm{~mm} \mathrm{Hg}$. The patient was extubated 7 hours post-delivery. Nebulized iloprost was initiated and allowed weaning of the nitric oxide. Heparin was initiated. Repeat Doppler echocardiography revealed that the RVSP was approximately $90 \mathrm{~mm} \mathrm{Hg}$. She was discharged from the hospital eleven days after arrival. Follow up in pulmonary hypertension clinic was arranged.

Primary Pulmonary Hypertension and Pregnancy ( $P$ $\mathrm{PH})$ : Pulmonary hypertension ( $\mathrm{PH})$ in pregnancy ( $\mathrm{P}-\mathrm{PH})$ is a high-risk medical condition. Women with primary pulmonary hypertension (PPH) should be strongly advised against pregnancy. In early pregnancy, there should be a careful discussion regarding termination because of the high risk to the fetus and mother [8]. Hemodynamic deterioration typically occurs in the second trimester with symptoms of dyspnea, syncope, and chest pain. This symptomatic period corresponds to the physiologic increase in cardiac output and blood volume of $40 \%$. When $\mathrm{PPH}$ is not diagnosed until late in pregnancy an elective delivery with caesarian section is preferred. Delivery should be planned for 32-34 weeks of gestation. An opiate based general anesthetic is considered appropriate for a failing RV. Additionally, the use of pulmonary vasodilators such as oxygen, nitric oxide, and iloprost are recommended. A multidisciplinary approach between obstetrics, cardiology, and anesthesia is of utmost importance for a successful maternal-fetal outcome. PPH complicating pregnancy remains a fatal condition with deaths occurring between 2 - 9 days post-delivery, usually from RV failure. lloprost and nitric oxide therapies may have a role in controlling PAP, but there is no evidence of increased survival [24].

\section{Take home message for P-PH}

- Mortality is high in women with P-PH. The maternal mortality ranges from $16-30 \%$. This mortality risk results in a recommendation to avoid pregnancy in patients with PPH and when pregnancy occurs in $\mathrm{PPH}$ patients the recommendation is for early termination of the pregnancy. The risk is greatest in the period from delivery of the placenta to early post-partum; typically, 6 weeks. The common causes of death are $\mathrm{PH}$ crisis, pulmonary thrombosis, and right HF. There is up to $30 \%$ fetal and neonatal death. Predictors include pre-term delivery, right HF, and hypoxia [8].

- A multidisciplinary team is required when caring for P-PH patients. Follow up must be stringent and in the third trimester should be weekly with an assessment including oxygen saturation and assessment of RV function. It is important to stop Bosentan and endolethin receptor antagonists if clinically feasible because of the association with embryopathy [8].
- The multidisciplinary team should strategize regarding optimal mode and timing of delivery. There should be development of a meticulous delivery blueprint. Regional anesthesia is preferred to general anesthesia, but is determined on a case-by-case basis. Meticulous fluid balance and optimization of RV function is critical because they play a role in determining clinical outcomes. $\mathrm{P}-\mathrm{PH}$ patients remain high-risk post-delivery and the mother and baby need careful and meticulous follow up medical care [8].

\section{Case 5}

A 27-year-old Caucasian female presented to our emergency department (ED) 4 days after giving birth. She reported being very short of breath for the last two days. She was unable to lie flat at night. Shortness of breath manifested as a respiratory rate of 20 breaths per minute; use of accessory muscles of respiration, and oxygen saturation of $81 \%$ on room air.

She had previously had good health except for developing gestational hypertension during her last month of pregnancy and dependent peripheral edema. She was treated with labetalol $100 \mathrm{mg}$ orally, twice daily, for blood pressure management.

In the emergency department her blood pressure was $160 / 90 \mathrm{~mm} \mathrm{Hg}$, pulse 95 beats per minute, respiratory rate 22 breaths per minute, and oxygen saturation 95\% on 2 liters of oxygen with nasal cannula. Auscultation revealed a S3. Lungs were clear to auscultation. There was mild peripheral edema, but no calf tenderness. D-Dimer was normal and BNP was elevated to 2000 pg/ $\mathrm{ml}$. ECG revealed sinus rhythm. Chest X-ray revealed cardiomegaly with increased vascular congestion. A computed tomography scan revealed no pulmonary emboli. Echocardiography revealed an ejection fraction of $35 \%$ with trace mitral regurgitation. The patient was admitted and treated with diuretic, labetalol, and lisinopril. She improved clinically. At follow up six months later, a repeat echocardiogram revealed normalization of LV systolic function.

Pregnancy and peripartum cardiomyopathy (P-PPCM): Peripartum cardiomyopathy (PPCM) is associated with one in every 3000 to 4000 live births in the United States [25]. The definition of PPCM includes 4 criteria: development of HF in the last month of pregnancy; or within 5 months of delivery, absence of an alternative identifiable cause of $\mathrm{HF}$, absence of recognizable heart disease before the last month of pregnancy, and LV ejection fraction less than 45\% [26]. Mortality is as high as $25 \%$ [27].

Clinical features of PPCM include symptoms of HF. Signs include tachycardia, tachypnea, pulmonary rales, and S3. BNP may be elevated. ECG may be normal. Chest $X$-ray typically has an enlarged cardiac silhouette and 
pulmonary congestion. Echocardiography is crucial to the diagnosis, often revealing cardiac enlargement and depressed LV systolic function [28]. Treatment is similar to other forms of $\mathrm{HF}$ with salt restriction, diuretic, betablocker, and digoxin. Hydralazine can be used during pregnancy to reduce afterload.

In approximately $50 \%$ of patients the LV ejection fraction normalizes. Despite recovery of ventricular function, a second pregnancy is not recommended because greater than $30 \%$ of PPCM patients recur, putting the baby and mother at risk. During the period of depressed LV systolic function, the mother is at risk for rhythm disturbances. Ventricular arrhythmias should trigger an electrophysiology consult for advice on the different therapeutic approaches based upon the structure and function of the heart [8].

\section{Take home message P-PPCM}

- PPCM has predisposing influences including multiparity, African ethnicity, pre-eclampsia, malnutrition, smoking, diabetes, advanced age, and teenage pregnancy. The trigger of PPCM is enigmatic. Theorized etiologies include inflammatory states and abnormalities in angiogenesis prompting vascular damage. The biologically active $16 \mathrm{kDa}$ prolactin and soluble fms tyrosine kinase 1 may initiate and promote PPCM [8].

- The diagnosis of PPCM is one of exclusion. Typically, it presents as HF secondary to LV systolic dysfunction toward the end of pregnancy and in the 6 months following delivery. The majority of PPCM cases are diagnosed post-partum. The LV cavity may or may not be dilated, but LVEF is typically $<45 \%$. Patients also can present with ventricular arrhythmias and/or cardiac arrest [8].

- Echocardiography establishing LV cavity dilation, LVEF $<30 \%$, and RV dysfunction are associated with poorer outcomes. Even when LV function normalizes, counseling against future pregnancies is recommended due to the potential recurrence of PPCM with adverse fetal and maternal outcomes [8].

\section{Case 6}

A 29-yr-old female physician, with a BMI of 20 , gave a history of paroxysmal supraventricular tachycardia (SVT) from the age of $14 \mathrm{yr}$. She experienced an increase in frequency of symptoms during her pregnancy. Her pre-pregnant symptoms consisted of palpitations every couple month, lasting around $5 \mathrm{~min}$, but occasionally up to $20 \mathrm{~min}$, and terminated by breath holding. More serious symptoms such as syncope, dizziness, dyspnea, or chest pains did not occur. Electrocardiogram, 2-D echocardiogram, and the Holter did not reveal any abnormalities. LV systolic function was normal as measured by LVEF and strain.

Past medical history included removal of a cystic hemangioma from her posterior fossa 5 years before her pregnancy. This was a benign lesion, which was removed uneventfully, and MRI scans have shown no recurrence. Pheochromocytoma may be associated with this tumor and catecholamine levels were investigated to exclude this as a cause for the arrhythmias.

During pregnancy, these episodes increased in frequency, consisting of palpitations lasting $10 \mathrm{~min}$ and terminated by breath holding. Again, no serious symptoms occurred. A longer episode was documented on an ECG at 34 weeks gestation at the emergency

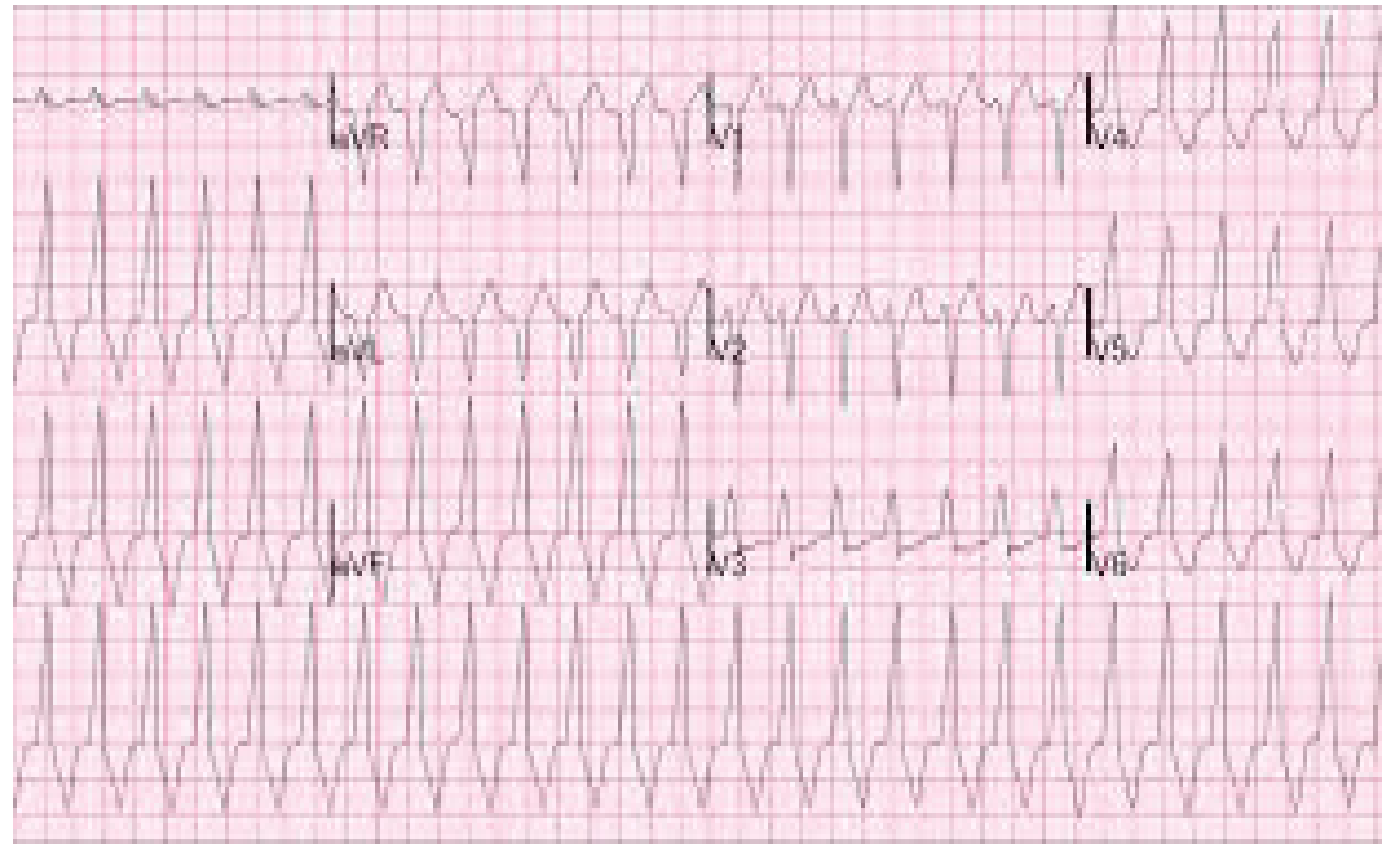

Figure 8: Wide complex tachycardia with LBBB and inferior axis. 
department revealing wide complex tachycardia at a rate of 160 beats per minute (Figure 8). During this episode the patient was hemodynamically stable. She was started on Lopressor $50 \mathrm{mg} /$ day. Because of concerns that induction of labor may exacerbate her cardiac problems, it was decided to deliver her by Caesarean section.

Caesarean section took place two weeks later, under a combined spinal epidural anesthetic. A healthy male infant was delivered. Spinal related hypotension was prevented with an infusion of ephedrine $30 \mathrm{mg}$ and phenylephrine $400 \mathrm{mg}$ in $500 \mathrm{ml}$ of normal saline, which was commenced immediately after the spinal injection. Syntocinon 5 units were administered slowly after delivery without problem. No further episodes of ventricular tachycardia occurred either intra-operatively or in the immediate postoperative period and she was discharged home 3 days later. The patient followed up with the electrophysiology service to discuss ablation therapy versus continued medical therapy for her RVOT VT.

Cardiac ventricular arrhythmias and pregnancy (P-CVR): Rhythm disturbances are commonly encountered during pregnancy in women with and without structural heart disease. Arrhythmias may manifest for the first time in pregnancy, or pregnancy may prompt an escalation of rhythm disturbances in women with a known pre-existing rhythm disorder [29].

Women with established arrhythmias or underlying structural heart disease are at the highest risk of developing arrhythmias during pregnancy. Women with congenital heart disease are at particularly high risk for arrhythmias. Since arrhythmias are frequently associated with acquired or congenital structural heart disease, any pregnant patient with arrhythmias should be evaluated with an electrocardiogram and transthoracic echocardiogram [30,31].

Arrhythmogenesis in pregnancy has been ascribed to the hemodynamic, hormonal, and autonomic changes associated with the pregnant state. The exact mechanism is unknown. Palpitations occur frequently during pregnancy and the sensation of palpitations in the absence of arrhythmias may be related to the enhanced cardiac output, increase heart rate, decreased peripheral resistance, and increased stroke volume associated with the pregnant state [32].

Ventricular premature beats (VPBs) are frequently detected in pregnant women. VPBs produce few or no symptoms in the preponderance of pregnant females, although some patients may experience palpitations or lightheadedness. No therapy is required for VPBs in asymptomatic patients. Patients should be told that the VPBs are benign [33]. If patients are experiencing intolerable symptoms metoprolol can be used.

Ventricular tachycardia (VT) and fibrillation (VF) are rare in pregnancy. VT can occur in patients with and without structural heart disease. The risk is higher in the setting of structural heart disease. Structural disease such as HCM, PPCM, congenital heart disease and valvular disease can be associated with VT in pregnancy. Myocardial infarction may occur in pregnancy and may be complicated by VT or VF. Pregnant patients with ion channelopathies are also at risk of VT [34].

In pregnant patients without structural heart disease and VT, the VT is considered to be idiopathic. The most common form is idiopathic right ventricular outflow tract (RVOT) VT that manifests as LBBB and inferior axis. The left ventricular outflow tract (LVOT) VT is less common and manifests as LBBB and inferior axis. Idiopathic VT usually has a benign prognosis and RVOT VT responds well to beta-blockers and LVOT VT responds well to verapamil $[35,36]$. These idiopathic ventricular tachycardias are very susceptible to treatment with ablations by the electrophysiology team post-delivery.

Long QT syndrome has the highest risk for VT in the post-partum period. This may be related to the decrease in heart rate and associated increase in QT duration. These patients should be treated with betablocker therapy during pregnancy and in the postpartum period [37].

Acute treatment of sustained ventricular arrhythmias in pregnant patients is similar to that in non-pregnant women. The decision regarding drug therapy, electrical therapy, ablation and implantation of defibrillators requires careful coordination with the electrophysiology team. Expert electrophysiology advice is recommended for pregnant patients with ventricular arrhythmias [8].

\section{Take home message P-CVR}

- Ventricular arrhythmia disorders should be assessed with imaging pre-pregnancy and during pregnancy to determine if there is structural heart disease. PPCM should be considered if there is new onset VT during the last 6 weeks of pregnancy or in the post-partum period. The recommendation for the acute termination of VT follows the 2015 ESC guidelines for management of patient with ventricular arrhythmias [38].

- Determining prophylactic drug therapy is linked to whether there is underlying structural heart disease. If there is no structural heart disease then idiopathic RV outflow tachycardia is the most frequent VT type, which responds to treatment with beta-blockers, verapamil, and other antiarrhythmic drug therapy. If drug therapy is ineffective then catheter ablation may be considered. Patients with long QT syndrome (LQTS) and those with catecholaminergic polymorphic VT (CPT) should receive non-selective beta-blocker therapy throughout pregnancy and up to at least 40 weeks [8]. Implantable cardio- 
defibrillator implantation (ICD) should follow guidelines outlined for the general population [38].

\section{Case 7}

A 28-year-old woman, with a history of idiopathic dilated cardiomyopathy and severely reduced left ventricular ejection fraction, presented at 21 weeks of gestation. Her medical history was complicated by asthma, obesity, obstructive sleep apnea, diabetes, and active tobacco abuse. She had an implantable cardioverter-defibrillator that had been placed for primary prevention of sudden cardiac death. She was considered, upon risk assessment, at prohibitively high risk for adverse maternal and fetal outcomes. After discussion of the different approaches to her pregnancy, given her high-risk status, she adamantly refused termination of pregnancy despite extensive counseling.

She was hospitalized for congestive heart failure at 26 weeks, which was managed with in-hospital bed rest and medical therapy consisting of digoxin, carvedilol, hydralazine, and furosemide. She developed progressive heart failure and declining left ventricular systolic function (left ventricular ejection fraction declined from $25 \%$ to $10 \%$ ). At 28 weeks of gestation the baby was delivered by urgent cesarean section with an epidural and conscious sedation. A pulmonary artery catheter and arterial line were placed for hemodynamic monitoring. Of note, the wedge pressure was 28 $\mathrm{mm} \mathrm{Hg}$ at the beginning of the procedure. Her postpartum course was complicated by a transient ischemic attack. Subsequently she developed hemodynamically unstable ventricular tachycardia that degenerated into ventricular fibrillation that was unresponsive to medical and electrical therapy. The baby survived.

\section{Pregnancy in patients with known dilated cardiomyopathy (P-DCM)}

Pregnant women with DCM have conventionally been counseled to avoid pregnancy. This advice is based upon the data from post-partum cardiomyopathy and the observation that there are adverse fetal and maternal outcomes in this subset [39]. The increase in intravascular volume and cardiac output during pregnancy leads to a greater risk of complications for women with DCM, particularly in the third trimester when the hemodynamic burden is extreme. A prior history of cardiac events is highly predictive of pregnancy related cardiac complications [40].

Patient with known LVEF $<40 \%$ are at higher risk for adverse fetal and maternal outcomes. These patients should be counseled regarding all the adverse potential outcomes with pregnancy. In patients with class 2-3 HF symptoms and LVEF $<40 \%$ in the first and second trimester should be counseled about the risks of carrying the pregnancy to term [41].
Management of labor and delivery in DCM patients is complex and requires a multidisciplinary approach between obstetrics, anesthesiology, and cardiology. Patient should be monitored continuously throughout labor and delivery as well as the early post-partum period. This includes electrocardiographic monitoring and noninvasive blood pressure monitoring. Invasive monitoring should be decided on an individual basis and be related to the complexity of the patient's hemodynamic status [42].

Management of labor and delivery is a multidisciplinary approach and if and when $\mathrm{HF}$ becomes refractory to medical therapy and the patient is on the precipice of hemodynamic instability delivery should be strongly considered. When possible, delivery should be through a vaginal approach because of the blood loss associated with the caesarian section.

In pregnant women with DCM, clinical parameters, including NYHA functional class and LV systolic function, can be used to identify women at highest risk for cardiac complications during pregnancy. When compared with non-pregnant women, pregnancy seems to have a negative impact on the clinical course for women with DCM, at least over the short term. Fetal and/or neonatal complications are also increased in mothers with DCM, and the risk is magnified by the presence of both cardiac and obstetric issues.

\section{Take home message for P-DCM}

- Patients with DCM should avoid pregnancy due to high maternal and fetal risk.

- P-DCM may be the consequence of multiple etiologies that result in a dilated LV with reduced LV systolic function. Up to $50 \%$ of P-DCM are idiopathic., with up to $30 \%$ being familial. The most common etiologies are viral infections, drugs, and ischemia. Personal and family histories are crucial factors [8].

- Patient may present with P-DCM de novo. The distinguishing clinical symptoms between normal pregnancy and HF can be challenging. Call for careful physical examination. PPCM and DCM are distinct entities, but share genotypic features. Presentations have overlapping features and differentiation during pregnancy can be challenging even for experts. HF with DCM or PPCM can occur rapidly and guidelines for management of acute HF apply [43].

\section{Case 8}

A 29-year-old female, with gestational age of 35 weeks, presented to obstetrics clinic with acute shortness of breath, fever, and cough with yellowish sputum. Chest X-ray was consistent with pneumonia. History revealed preexistent cryptogenic cirrhosis and mild preeclampsia. She was being treated with 
methyldopa for her hypertension and antibiotics for her pneumonia. Cardiac examination revealed hyperdynamic precordium with a systolic ejection murmur.

Echocardiography revealed a bicuspid aortic valve with moderate $A R$, dilated aorta $(45 \mathrm{~mm})$ with normal ventricular function. Treatment with blood pressure medications, oxygen, antibiotics, bronchodilator, antipyretic, and diuretic were initiated. Further evaluation revealed normal liver enzymes and platelet count of $60,000 / \mathrm{mm}^{3}$, which improved to $100,000 /$ $\mathrm{mm}^{3}$ on platelet transfusion. However, on second day of admission patient decompensated with progressive shortness of breath and oxygen saturation of $89 \%$ with face mask at $\mathrm{FiO} 2$ of $40 \%$. No features of fetal distress were evident. Emergent caesarean section delivery was planned.

She had a pulmonary artery catheter and arterial line inserted for continuous hemodynamic monitoring throughout the delivery and early post-partum period. There was continuous ECG and oxygen saturation monitoring throughout the delivery. She had no hemodynamic instability or arrhythmias. The postoperative period was uneventful. She was discharged ambulatory from the hospital on the $6^{\text {th }}$ post-operative day.

Bicuspid aortic valve with associated dilated aorta and pregnancy (P-BAV-aortopathy): Women with bicuspid aortic valve (BAV) should be counseled about the potential risks and treatments prior and during pregnancy. Potential risks include heritable congenital heart disease, aortic root enlargement with potential for dissection, and the aortic valve issues including aortic stenosis and/or aortic regurgitation.

Pregnant women with a BAV and aortic root dilation are at risk for spontaneous aortic dissection, usually in the third trimester and this is especially pertinent if there is coarctation [44]. Pregnancy associated increase in heart rate, blood pressure, and cardiac output result in increased aortic root stress and may play a role in promoting aortic root dilation and dissection. For women planning pregnancy prophylactic aortic root / ascending aorta surgery should be discussed if the aortic diameter is greater than $45 \mathrm{~mm}$, if the rate of increase in the diameter is $>5 \mathrm{~mm} /$ year, and if there is moderate or severe aortic regurgitation [42]. All pregnant women with thoracic aortic dilation should maintain strict blood pressure control with goal being 130/80. What is unknown is whether pregnancy changes the natural history of BAV with aortopathy as demonstrated in Marfan syndrome $[45,46]$.

BAV that is stenotic poses more complexity to pregnancy than a BAV that is regurgitant. All women with symptomatic BAV stenosis and asymptomatic patients with high-risk features, such as severe aortic stenosis and LVEF $<40 \%$, should delay conception until the aortic stenosis has been managed surgically or through percutaneous approaches. Moderate to severe aortic regurgitation with preserved LVEF is usually well tolerated in pregnancy [47].

Surgery should be avoided during pregnancy. Although, if clinical circumstances dictate a lifesaving surgical intervention may be indicated if valvotomy is not an option. Controlled vaginal delivery is preferable, but caesarian section is reasonable in the presence of severe cardiovascular diseases such as aortic dissection, severe aortic stenosis, and in the setting of anticoagulation.

\section{Take home message P-BAV-aortopathy}

- P-BAV-Aortopathy patients should be monitored meticulously throughout pregnancy and for 6 months post-partum. For patients in this subset with a high risk for dissection or a dilated aorta, monthly monitoring with imaging is reasonable. Lower risk patients should be monitored every 12 weeks. Strict blood pressure control is prudent [8].

- Progressive root dilation during pregnancy, before the fetus is viable, may require surgical intervention with the fetus in utero. When the fetus is viable caesarian delivery should occur followed by aortic surgery. Stanford type A dissection during pregnancy is a surgical emergency. A multidisciplinary team of cardiology, CV surgery, obstetrics and anesthesiology must conference and proceed directly to surgery. Although maternal outcome is good, fetal mortality approaches $30 \%$. Uncomplicated type B dissection demands good blood pressure control. Complicated type B dissection may require surgery [8].

- Delivery requires a multidisciplinary team approach and if the ascending aorta is $40-45 \mathrm{~mm}$ on an individualized basis vaginal and caesarian delivery can be considered. Caesarian delivery is most prudent if the aorta is $>45 \mathrm{~mm}$ [8].

\section{Conclusion}

This manuscript attempts to introduce the readership to some interesting cardiovascular conditions that surface during pregnancy. Hopefully the cases act as a stimulus to reading the comprehensive 2018 ESC guidelines for the management of cardiovascular disease during pregnancy. The guidelines provide an exhaustive review of the fascinating cardiovascular conditions that physicians may encounter when caring for patients during pregnancy.

\section{References}

1. Maeder M, Amman P, Angehrn W, Hans Rickli (2005) 
Idiopathic spontaneous coronary artery dissection: Incidence, diagnosis and treatment. Int J Card 101: 363369.

2. De Maio SJ JR, Kinsella SH, Silverman ME (1989) Clinical course and long-term prognosis of spontaneous coronary artery dissection. Am J Cardiology 64: 471-474.

3. Heefner WA (1973) Dissecting hematoma of the coronary artery. A possible complication of oral contraceptive therapy. JAMA 223: 550-551.

4. Bonnet J, Aurmailley M, Thomas D, Grosgogeat $Y$, Broustet JP, et al. (1986) Spontaneous coronary artery dissection: Case report and evidence for a defect in collagen metabolism. Eur Heart J 7: 904-909.

5. Judge JP, Dietz HC (2005) Marfan's syndrome. Lancet 336: 1965-1976.

6. Ades LC, Waltham RD, Chiodo AA, Bateman JF (1995) Myocardial infarction resulting from coronary artery dissection in an adolescent with Ehlers-Danlos 4 due to type 3 collagen mutation. Br Heart J 754: 112-116.

7. Kamineni R, Sadhu S, Alpert JS (2002) Spontaneous coronary artery dissection: Report of two cases and a 50year review of the literature. Cardiol Rev 10: 279-84.

8. Vera Regitz-Zagrosek, Jolien W Roos-Hesselink, Johann Bauersachs, Carina Blomström-Lundqvist, Renata Cífková, et al. (2018) 2018 ESC Guidelines for the management of cardiovascular disease during pregnancy. Eur Heart $\mathrm{J} 39$ : $3165-3241$.

9. Akashi YJ, Goldstein DS, Barbaro G, Ueyama T (2008) Takotsubo cardiomyopathy: A new form of acute, reversible heart failure. Circulation 118: 2754-2762.

10. Bybee KA, Kara T, Prasad A, Lerman A, Barsness GW, et al. (2004) Systematic review: Transient left ventricular apical ballooning: A syndrome that mimics ST-segment elevation myocardial infarction. Ann Intern Med 141: 858-865.

11. Lyon AR, Rees PS, Prasad S, Poole-Wilson PA, Harding SE (2008) Stress (Takotsubo) cardiomyopathy--a novel pathophysiological hypothesis to explain catecholamine induced acute myocardial stunning. Nat Clin Pract Cardiovasc Med 5: 22-29.

12. Sharkey SW, Lesser JR, Maron MS, Maron BJ (2011) Why not just call it tako-tsubo cardiomyopathy: A discussion of nomenclature. J Am Coll Cardiol 57: 1496-1497.

13. Song BG, Park SJ, Noh HJ, Jo HC, Choi JO, et al. (2010) Clinical characteristics, and laboratory and echocardiographic findings in takotsubo cardiomyopathy presenting as cardiogenic shock. J Crit Care 25: 329-335.

14. Kapoor D, Bybee KA (2009) Stress cardiomyopathy syndrome: A contemporary review. Curr Heart Fail Rep 6: 265-271.

15. Cherian J, Angelis D, Filiberti A, Saperia G (2007) Can takotsubo cardiomyopathy be familial? Int $\mathrm{J}$ Cardiol 121: 74-75.

16. Hurst RT, Prasad A, Askew JW 3rd, Sengupta PP, Tajik AJ (2010) Takotsubo cardiomyopathy: A unique cardiomyopathy with variable ventricular morphology. JACC Cardiovasc Imaging 3: 641-649.

17. Christian Templin, Jelena Ghadri (2015) Clinical Features and Outcomes of Takotsubo (Stress) Cardiomyopathy. NEJM Sept 373.

18. Toglia MR, Weg JG (1996) Venous thromboembolism during pregnancy. NEJM 335: 108-114.
19. Epiney M, Boehlen F, Boulvain M, Reber G, Antonelli $\mathrm{E}$, et al. (2005) D-dimer levels during delivery and the postpartum. J Thromb Haemost 3: 268-271.

20. Arnaud P, Roy PM, Sanchez O, Gal GL, Meyer G, et al. (2005) Multidetector computed tomography in suspected pulmonary embolism. N Engl J Med 352: 1760-1768.

21. Rogers M, Well PS (2001) Diagnosis Thrombosis Research 103: v225-v238.

22. (2000) Guidelines on diagnosis and management of acute pulmonary embolism, task force on pulmonary embolism, European Society of Cardiology. Eur Heart J 21: 13011336.

23. What is Amniotic Fluid Embolism?

24. Anna R Hemnes, David G Kiely, Barbara A Cockrill, Zeenat Safdar, Victoria J Wilson, et al. (2015) Statement on pregnancy in pulmonary hypertension from the pulmonary vascular Research Institute. Pulm Circ 5: 435-465.

25. Libby P, Bonow RO, Mann DL, Zipes DP (2007) Braunwald's heart disease: A textbook of cardiovascular medicine. $\left(8^{\text {th }}\right.$ edn), Elsevier Science, Philadelphia.

26. Pearson GD, Veille JC, Rahimtoola S, Hsia J, Oakley CM, et al. (2000) Peripartum cardiomyopathy: National Heart, Lung, and Blood Institute and Office of Rare Diseases (National Institutes of Health) workshop recommendations and review. JAMA 283: 1183-1188.

27. Abboud J, Murad Y, Chen-Scarabelli C, Saravolatz L, Scarabelli TM (2007) Peripartum cardiomyopathy: A comprehensive review. Int J Cardiol 118: 295-303.

28. Davidson NM, Parry EH (1979) The etiology of peripartum cardiac failure. Am Heart J 97: 535-536.

29. Li JM, Nguyen, Joglar JA, Hamdan MH, Page RL, et al. (2008) Frequency and outcome of arrhythmias complicating admission during pregnancy: Experience form a highvolume and ethnically-diverse obstetric service. Clinical Cardiol 31: 538-541.

30. Siu SC, Sermer M, Colman JM, Alvarez AN, Mercier LA, et al. (2001) Prospective multicenter study of pregnancy outcomes in women with heart disease. Circulation 104: 515-521.

31. Drenthen W, Pieper PG, Roos-Hesselink JE, Van Lottum WA, Voors AA, et al. (2007) Predictors of pregnancy complications in women with congenital heart disease. $\mathrm{J}$ Am Coll Cardiol 49: 2303-2311.

32. Silversides CK, Colman JM (2007) Physiologic changes in pregnancy. In: Celia Oakley, Carole A Warnes, Heart disease in pregnancy. ( $2^{\text {nd }}$ edn), Blackwell Publishing, Malden.

33. Shotan A, Ostrzega E, Mehra A, Johnson JV, Elkayam $U$ (1997) Incidence of arrhythmias in normal pregnancy and relation to palpitations, dizziness and syncope. Am J Cardiol 79: 1061-1064.

34. Zipes DP, Camm AJ, Borggrefe M, Buxton AE, Chaitman $B$, et al. (2006) ACC/AHA/ESC 2006 Guidelines for Management of Patients with Ventricular Arrhythmias and the Prevention of Sudden Death: A report of the American College of Cardiology/ American Heart Association Task Force and the European Society of Cardiology Committee for Practice Guidelines: Developed in collaboration with the European Heart Rhythm Association and the Heart Rhythm Society. Circulation 114: e385-e484.

35. Lin Fc, Finley CD, Rahimtoola SH, Wu D (1983) Idiopathic paroxysmal ventricular tachycardia with a QRS pattern of 
right bundle branch block and left axis deviation: A unique clinical entity with specific properties. Am J Cardiol 52: 95100.

36. J Cleary-Goldman, C R Salva, J I Infeld, J N Robinson (2003) Verapamil-sensitive idiopathic left ventricular tachycardia in pregnancy. J Maternal Fetal Neonatal Med 14: $132-135$

37. Rashba EJ, Zareba W, Moss AJ, Hall W J, Robinson J, et al. (1998) Influence of pregnancy on the risks for cardiac events with heredity long QT syndrome. LQTS investigators. Circulation 97: 451-456.

38. Silvia G Priori, Carina Blomström-Lundqvist, Andrea Mazzanti, Nico Blom, Martin Borggrefe, et al. (2015) 2015 ESC guidelines for the management of patients with ventricular arrhythmias and the prevention of sudden cardiac death: The task force for the management of patients with ventricular arrhythmias and the prevention of sudden cardiac death of the European Society of Cardiology (ESC). Endorsed by: Association for European Paediatric and Congenital cardiology (AEPC) 2015 ESC guidelines for the management of patients with ventricular arrhythmias and the prevention of sudden cardiac death: The task force for the management of patients with ventricular arrhythmias and the prevention of sudden cardiac death of the European Society of Cardiology (ESC). Endorsed by: Association for European Paediatric and Congenital cardiology (AEPC). Eur Heart J 36: 2793-2867.

39. S C Siu, M Sermer, D A Harrison, E Grigoriadis, G Liu, et al. (1997) Risks and predictors for pregnancy-related complications in women with heart disease. Circulation 96: 2789-2794.

40. Samuel C Siu, Jack M Colman, Sheryll Sorensen, Jeffrey F Smallhorn, Dan Farine, et al. (2002) Adverse neonatal and cardiac outcomes are more common in pregnant women with cardiac disease. Circulation 105: 2179-2184.
41. Jasmine Grewal, Samuel C Siu, Heather J Ross, Jennifer Mason, Olga H Balint, et al. (2009) Pregnancy outcomes in in women with dilated cardiomyopathy. J Am Coll Cardiol 55: 45-52.

42. Jonathan G Howlett, Robert S McKelvie, Jeannine Costigan, Anique Ducharme, Estrellita Estrella-Holder, et al. (2010) The 2010 Canadian cardiovascular society guidelines for the diagnosis and management of heart failure update: heart failure in ethnic minority populations, heart failure and pregnancy, disease management, quality improvement/ assurance programs. Can J Cardiol 26: 185-202.

43. Piotr Ponikowski, Adriaan A Voors, Stefan D Anker, Héctor Bueno, John G F Cleland, et al. (2016) 2016 ESC guidelines for the diagnosis and treatment of acute and chronic heart failure: The task force for the diagnosis and treatment of acute and chronic heart failure of the European Society of Cardiology (ESC) developed with the special contribution of the Heart Failure Association (HFA) of the ESC. Eur Heart J 37: 2129-2200.

44. Anderson RA, Fineron PW (1994) Aortic dissection in pregnancy; importance of pregnancy- induced changes in the vessel wall and bicuspid valve in pathogenesis. $\mathrm{Br} J$ Obstet Gynaecol 101: 1085-1088.

45. Rick A Nishimura, Catherine M Otto, Robert O Bonow, Blase A Carabello, John P Erwin 3rd, et al. (2014) 2014 AHA/ACC guidelines for the management of patients with valvular heart disease: A report of the American college of cardiology/American heart association task force on practice guidelines. J Am Coll Cardiol 63: e57-e185.

46. Ryan T Donnelly, Nelangi M Pinto, Irene Kocolas, Anji T Yetman (2012) The immediate and long-term impact of pregnancy on aortic growth rate and mortality in women with marfan syndrome. J Am Coll Cardiol 60: 224-229.

47. (2004) Expert consensus document on management of cardiovascular diseases during pregnancy. Eur Heart J 24: 761-781. 